\title{
Strongly driven surface-global Kinetic Ballooning Modes in general toroidal geometry
}

\author{
A Zocco ${ }^{1}$, K Aleynikova ${ }^{1,2}, \mathbf{P}$ Xanthopoulos ${ }^{1}$ \\ ${ }^{1}$ Max-Planck-Institut für Plasmaphysik, D-17491, Greifswald, Germany \\ ${ }^{2}$ Moscow Institute of Physics and Technology, Dolgoprudny, Russian Federation
}

(Received 23 May 2018)

Kinetic Ballooning Modes in magnetically confined toroidal plasmas are investigated putting emphasis on specific stellarator features. In particular, we propose a Mercier criterion which is purposely designed to allow for direct comparison with local flux-tube gyrokinetics simulations. We investigate the influence on the marginal frequency of the mode of a magnetic curvature which is inhomogeneous on the magnetic flux surface due to the field-line-label dependence. This is a typical (surface) global effect present in nonaxisymmetry. Finally, we propose an artificial equilibrium model that explicitly retains the field-line-label dependence in the magnetic drift, and analyse the stability of the system by introducing a representation of the perturbations similar to the flux-bundle model of Sugama et al. [Plasma and Fusion Research, 7, 2403094 (2012)]. The coupling of flux-bundles is shown having a stabilizing effect on the most unstable local flux-tube mode.

\section{Introduction}

In recent years, great effort has been devoted to the investigation of gyrokinetic instabilities that can cause turbulent transport in stellarators. In particular, analytical and numerical progress has been made for electrostatic instabilities, such as trapped electron modes (Proll et al. 2013; Faber et al. 2015), ion-temperature-gradient driven modes (Plunk et al. 2014; Helander et al. 2015; Zocco et al. 2016; Xanthopoulos et al. 2016) and electron-temperature-gradient driven modes (Jenko \& Kendl 2002). Electromagnetic gyrokinetic instabilities have been explored much less. At present, our understanding is based on the use of numerical codes and is limited to a handful of works (Sugama \& Watanabe 2004; Baumgaertel et al. 2012; Ishizawa et al. 2015; Mishchenko et al. 2015; Ishizawa et al. 2014). This status quo is clearly not satisfactory, especially if we consider our lack of analytical insight. The state of affairs is different in the sphere of energetic particles physics, especially in tokamaks, where there is certainly no lack of analyticallydriven research [see the review of Chen \& Zonca (2016) and references therein]. For transport studies, a first step towards the reconciliation of analytics and numerics, for strongly-driven kinetic ballooning modes (KBMs), was made in the work of Aleynikova \& Zocco (2017). Here, quantitative agreement between electromagnetic gyrokinetic numerical simulations and a finite- $\beta$ (where $\beta$ is the ratio of kinetic to magnetic plasma pressure) diamagnetic modification of ideal MHD was found. The results of Aleynikova \& Zocco (2017), however, only apply to a simple geometric setting, and an extension to more relevant geometries is required. In this article we complement the numerical work of Aleynikova et al. (2018) on the stellarator Wendelstein 7-X, and put forward an analytical formulation of the diamagnetic modification of ideal MHD used by Aleynikova \& Zocco (2017) in a surface-global setting. Our analysis will then be local in the radial direction of the torus, but the equilibrium magnetic field is allowed to vary on the 
magnetic flux surface with respect to the field line as is possible in non-axisymmetric geometries. We show how to properly choose co-ordinates in such a way that the original derivation of the Mercier criterion (Mercier 1960; Mercier \& Luc 1974) can be performed also in stellarator geometry with the use of the ballooning transform of Connor et al. (1979). We identify the metric elements that characterize surface-global effects and study how they impact the real frequency of the diamagnetically modified ideal MHD mode proposed by Aleynikova \& Zocco (2017). Finally, a discrete description similar to the flux-bundle model of Sugama et al. (2012) is introduced. The effect of the field-line-label dependence on the curvature drift is investigated within this framework and it is found to be stabilising. This stabilisation is related to a possible violation of the Mercier criterion.

\section{Formulation}

The equation for the divergence of the plasma current, when each term is ordered to accommodate linear ballooning modes, results in a second order differential equation, in the field-line following variable $l$, for the potential $\psi$ that defines the parallel component of the magnetic potential $-i \omega A_{\|}=\nabla_{\|} \psi$. Here $\omega$ is the complex mode frequency, $\nabla_{\|}=\mathbf{b} \cdot \nabla$, with $\mathbf{b}=\mathbf{B} / B$, where $\mathbf{B}$ is the equilibrium magnetic field, and $B$ is its modulus. The general form of this equation is Eq. (2.35) of Tang et al. (1980). This comes from a sound expansion of the gyrokinetic equation, $k_{\|} v_{t h i} \ll \omega \ll k_{\|} v_{t h e}$. Here $v_{t h s}=\sqrt{2 T_{s} / m_{s}}$ is the thermal speed for a species with temperature $T_{s}$ and mass $m_{s}$. When a finite $\beta \sim \epsilon \equiv$ $k_{\|}^{2} v_{t h i}^{2} / \omega^{2} \ll 1$ ordering is implemented, magnetic compressibility is retained, and the curvature and grad- $B$ drifts are kept consistent with the Grad-Shafranov equation, the relevant equation for kinetic ballooning modes is a simple diamagnetic modification of the ideal MHD ballooning equation (Roberts \& Taylor 1965; Aleynikova \& Zocco 2017)

$$
\frac{B / B_{a}^{2}}{\beta_{i}} \frac{v_{t h i}^{2}}{\omega^{2}} \nabla_{\|} b B \nabla_{\|} \psi=-b\left[1-\frac{\omega_{* i}}{\omega}\left(1+\eta_{i}\right)\right] \psi-2 \frac{\omega_{\kappa} \omega_{p}}{\omega^{2}} \psi,
$$

where $B_{a}$ is a reference constant magnetic field, $\beta_{i}=8 \pi p_{i} / B_{a}^{2}, b=0.5 k_{\perp}^{2} v_{t h i}^{2} / \Omega_{i}(B)^{2}, \mathbf{k}_{\perp}$ is the wave vector (of perturbations) across the equilibrium magnetic field, and $\Omega_{i}(B)=$ $m_{i} c /(e B)$ is the ion cyclotron frequency. In a surface-global setting, the $\mathbf{k}_{\perp}^{2}=k_{i} k^{i}=$ $k^{j} g_{j i} k^{i}$ term becomes the Laplacian operator in curvilinear geometry

$$
b=-\frac{1}{2} \frac{\rho_{i}^{2}}{a^{2}} \frac{B_{a}^{2}}{B^{2}} \frac{1}{\sqrt{g}} \sum_{i, j=1}^{2} \frac{\partial}{\partial x^{i}} \sqrt{g} g^{i j} \frac{\partial}{\partial x^{j}},
$$

where $\rho_{i}=v_{t h i} / \Omega_{i}\left(B_{a}\right)$, and we introduced a triplet of contravariant co-ordinates $\mathbf{x}=$ $\left(x^{1}, x^{2}, x^{3}\right)$. Each $x^{i}$ is a scalar function of the Cartesian spatial co-ordinates $(x, y, z)$. We then define the contravariant metric tensor $g^{i j}=\nabla x^{i} \cdot \nabla x^{j}$ where $\nabla=\mathbf{e}^{x} \partial_{x}+\mathbf{e}^{y} \partial_{y}+\mathbf{e}^{z} \partial_{z}$ is the gradient in Cartesian co-ordinates, $\sqrt{g}=\left(\nabla x^{1} \times \nabla x^{2} \cdot \nabla x^{3}\right)^{-1}$ is the determinant of the Jacobian matrix $J_{i}^{j}=\partial_{i} x^{j}$, and $a$ is a reference length scale. The functions $g^{i j}$ will soon be specified. The diamagnetic frequency is

$$
\omega_{* s}=\frac{1}{2} \frac{v_{t h s}}{L_{n}} \frac{\rho_{s}}{a}\left(-i \frac{\partial}{\partial x^{2}}\right)
$$

where $L_{n}^{-1}=d \ln n / d x^{1}$. Then $\omega_{p s}=\omega_{* s}\left(1+\eta_{s}\right)$, with $\eta_{s}=d \ln T_{s} / d \ln n, \omega_{p}=\omega_{p i}-\omega_{p e}$, and $L_{p, s}^{-1}=L_{n}^{-1}\left(1+\eta_{s}\right)$, with $L_{p}^{-1}=L_{p, i}^{-1}+L_{p, e}^{-1}$. Notice that we have corrected a multiplicative factor 2 on the LHS of Eq. (2.1) of Aleynikova \& Zocco (2017). We will now be more specific with the co-ordinate system.

We follow Xanthopoulos et al. (2009) and consider a modification of the Boozer system 
(Boozer 1982) that respects the field alignment:

$$
\left(x^{1}, x^{2}, x^{3}\right)=\left(s, q(s)\left(\theta-\theta_{0}\right)-\zeta, \theta-\theta_{0}\right),
$$

where $s=\Phi / \Phi_{\text {edge}}$, with $\Phi$ the toroidal magnetic flux and $\Phi_{\text {edge }}$ its value at the last closed flux surface, and $\theta$ and $\zeta$ are the Boozer poloidal and toroidal angles, respectively. Thus, $\sqrt{g_{B}} B^{2}=B_{\theta} \Psi^{\prime}(s)+B_{\zeta} \Phi^{\prime}(s)$, where $\Psi$ is the poloidal magnetic flux, and $\mathbf{B}=$ $\Psi^{\prime}(s) \nabla x^{1} \times \nabla x^{2}$. The prime is a total derivative with respect to the explicit argument $s, q=\Phi^{\prime} / \Psi^{\prime} \equiv \iota^{-1}$, and $\theta_{0}$ is the familiar free parameter of ballooning theory (Connor et al. 1978, 1979; Hastie \& Taylor 1981). We have

$$
\begin{aligned}
& \omega_{\kappa}=-i \mathbf{v}_{d} \cdot \nabla \\
& =-i v_{t h i}^{2} \frac{B_{a}}{B} \frac{\hat{\mathbf{b}} \times \kappa}{\Omega_{i}\left(B_{a}\right)} \cdot \nabla \\
& =-i v_{t h i} \rho_{i} \frac{B_{a}}{B} \sum_{i=1}^{2} \hat{\mathbf{b}} \times \kappa \cdot \nabla x^{i} \frac{\partial}{\partial x^{i}},
\end{aligned}
$$

where $\kappa=\hat{\mathbf{b}} \cdot \nabla \hat{\mathbf{b}}$. We now introduce our first assumption: $\partial_{x^{1}} \equiv 0$. That is, we are neglecting the radial structure of the mode under consideration. From this, it also follows that $\theta_{0} \equiv 0$, since in ballooning theory it can be shown that $\theta_{0}$ is proportional to the radial wave number. The effect of $k_{1}$ must be carefully considered for each non-axisymmetric machine under consideration, depending on the global shear of its configurations. For instance, a finite $k_{1}$ seems to be crucial to capture the most unstable KBM in LHD [see Fig. (1) of Ishizawa et al. (2014)]. In the case of W7-X, $k_{1}$ could have a less prominent role (Aleynikova et al. 2018).

By using Eq. (23) of Xanthopoulos et al. (2009), and the properties of Boozer coordinates, after some straightforward algebra, we obtain

$$
\omega_{\kappa} \omega_{p}=-\frac{1}{4} \frac{\rho_{i}^{2}}{a^{2}} \frac{v_{t h i}^{2}}{L_{p} a}\left[B_{s} B \nabla_{\|}\left(\frac{1}{B^{2}}\right)+\frac{P^{\prime}(s)}{B^{2} / 2}+\frac{\partial_{s} B^{2}}{B^{2}}-\frac{a^{2} B_{a} B}{2 P^{\prime}(s)} \nabla_{\|}\left(\frac{j_{\|}}{B}\right) \hat{s} \theta\right] \frac{\partial^{2}}{\partial\left(x^{2}\right)^{2}},
$$

where $j_{\|}=\hat{\mathbf{b}} \cdot \mathbf{j}$, j is the plasma current, and $\hat{s}=2 s_{0} q^{\prime}\left(s_{0}\right) / q\left(s_{0}\right)$ is the global magnetic shear at a given radial location $s_{0}$, and we used $\Psi_{N}^{\prime}=\Psi^{\prime} /\left(a^{2} B_{a}\right)=\sqrt{s} / q$ [see also Eq. (141) of Xanthopoulos et al. (2009)]. This form will be extremely important in order to derive a Mercier criterion, because of the explicit $\nabla_{\|}$. We finally choose $x^{3}=\theta$, so that $\sqrt{g_{N}} a \nabla_{\|}=\left(B_{a} / B\right) \partial_{\theta}$, where $\sqrt{g_{N}}=2 q a^{-3} \sqrt{g_{B}}$ is the normalised Jacobian.

It is now possible to specify the form of Eq. (2.2). The metric elements entering Eq. (2.2) have first been presented by Cooper (1992) and have also been evaluated by Xanthopoulos et al. (2009). Then, we find it convenient to write

$$
\begin{aligned}
b & =-\frac{1}{2} \frac{\rho_{i}^{2}}{a^{2}} \frac{B_{a}^{2}}{B^{2}} \frac{1}{\sqrt{g_{N}}} \frac{\partial}{\partial x^{2}} \sqrt{g_{N}} g_{B}^{22} \frac{\partial}{\partial x^{2}} \\
& =-\frac{1}{2} \frac{\rho_{i}^{2}}{a^{2}} \frac{B_{a}^{2}}{B^{2}} \frac{1}{\sqrt{g_{N}}} \frac{\partial}{\partial x^{2}} \sqrt{g_{N}}\left[b_{0}+b_{1} \hat{s} \theta+b_{2} \hat{s}^{2} \theta^{2}\right] \frac{\partial}{\partial x^{2}},
\end{aligned}
$$

with $b_{0}=\left(g_{s s} B^{2}-B_{s}^{2}\right) /\left(a^{2} B_{a}^{2}\right), b_{1}=\left(B_{\theta} B_{s}-g_{s \theta} B^{2}\right) /\left(s_{0} a^{2} B_{a}^{2}\right)$, and $b_{2}=g^{s s} a^{2} /\left(4 s_{0}\right) \equiv$ $g_{N}^{s s}$. Since we are assuming (for perturbations!) $\partial / \partial x^{1}=\theta_{0} \equiv 0$, the $g_{B}^{22}$ term is the only metric element left in the summation that defines $b$ in Eq. (2.2). It is perhaps interesting to note that, in Eq. (2.5), the function $b$ shows the same $\hat{s} \theta$ dependence that it would have in the well known $\hat{s}-\alpha$ model: $b_{\hat{s}-\alpha} \propto k_{2}^{2}+k_{1} k_{2} \hat{s} \theta+k_{2}^{2} \hat{s}^{2} \theta^{2}$. However now the coefficients $b_{i}$ are not constant, and we have a linear secular term even if $k_{1} \equiv 0$ ! This is 
purely geometric, and comes from the off-diagonal entries of the metric tensor. However, this term does not play a role in the formulation of the Mercier criterion.

\subsection{Local Mercier criterion and its validity}

Before analyzing the properties of Eq. (2.1) when the $x^{2}$-variation of the eigenfunction is allowed, it seems reasonable to follow the analysis of Connor Hastie and Taylor of the ballooning equation (Connor et al. 1979), and derive a Mercier criterion which is valid in a local flux-tube gyrokinetic context for stellarators. This is important since, historically, the derivation of the ballooning equation for stellarators has been based on a complicated minimisation of the ideal MHD pontential (Correa-Restrepo 1978) à la Mercier (Mercier 1960; Mercier \& Luc 1974) and its application to local flux-tube gyrokinetics is not straightforward. In some works on ideal MHD ballooning modes in stellarators, Hamada (1962) co-ordinates are used (Correa-Restrepo 1978). In others (Hegna \& Nakajima 1998), Boozer co-ordinates are introduced but the field-following co-ordinate is not specified and the secular terms are expressed implicitly in terms of integrals on the local shear. The "stellarator expansion" was used by Sugama \& Watanabe (2004). The most explicit formulation of the Mercier criterion for stellarators is the one given in a not so very accessible article by Nührenberg \& Zille (1987), where the authors use the toroidal angle as the field-following co-ordinate and do not order the global shear with the plasma $\beta$, unlike us. Additional instances of the use of a Mercier criterion in stellarators (Gardner \& Blackwell 1992; Fu et al. 1992) lead to Chapter 5 of the book of Bauer et al. (1984), which, in turns, leads cyclically to the work of Mercier \& Luc (1974). Since in this bibliographical Odyssey, lasting more than 38 years (therefore nearly 4 times the original Odyssey), we could not find a derivation of the indicial ballooning equation that: is based on the poloidal angle being the field-line-following co-ordinates, relates to local-flux-tube gyrokinetics, and gives an explicit ordering for the global shear, we decided to present such calculation here. The equation we study is then

$$
\begin{aligned}
& \frac{1}{\sqrt{g_{N}}} \frac{\partial}{\partial \theta} \frac{b}{\sqrt{g_{N}}} \frac{\partial}{\partial \theta} \psi=-b \frac{\omega\left(\omega-\omega_{p i}\right)}{\omega_{A}^{2}} \psi \\
& -\frac{\rho_{i}^{2}}{2 a^{2}} \frac{k_{2}^{2} v_{t h i}^{2}}{a L_{p} \omega_{A}^{2}}\left\{\frac{B_{s}}{B_{a}} \frac{1}{\sqrt{g_{N}}} \frac{\partial}{\partial \theta} \frac{B_{a}^{2}}{B^{2}}+2 \frac{P^{\prime}(s)}{B^{2}}+\frac{\partial_{s} B^{2}}{B^{2}}-\frac{a B_{a}^{2}}{P^{\prime}(s)} \frac{1}{\sqrt{g_{N}}} \frac{\partial}{\partial \theta}\left(\frac{j_{\|}}{B}\right) \hat{s} \theta\right\} \psi,
\end{aligned}
$$

with $\omega_{A}^{2}=v_{t h i}^{2} /\left(\beta_{i} a^{2}\right), b=\rho_{i}^{2} k_{2}^{2} B_{a}^{2} /\left(2 a^{2} B^{2}\right) g_{N}^{22}$, and $g_{N}^{22}=b_{0}+b_{1} \hat{s} \theta+b_{2} \hat{s}^{2} \theta^{2}$, where the $b_{i}$ have been defined in the previous section.

We are now in the position to seek a solution of the type

$$
\psi=z^{\alpha}\left(g_{0}+\frac{g_{1}}{z}+\frac{g_{2}}{z^{2}}+\ldots\right)
$$

where $z=\hat{s} \theta$, and we consider radial locations for which $\iota=n / m$, with $m$ and $n$ integers. Then, the functions $g_{i}$ have the period of the equilibrium, and $\int_{\Gamma} d g_{i}=0$ if $\Gamma$ in the path on integration along a closed field line. When the field line is chosen to be a highorder rational $\int_{\Gamma}(\cdots) d \theta / \int_{\Gamma} d \theta \approx(2 \pi)^{-2} \int_{0}^{2 \pi} d \theta \int_{0}^{2 \pi} d \zeta(\cdots)$. The index $\alpha$ is a complex quantity which determines a necessary condition for marginal stability. Rigorously, the diamagnetic correction of Eq. (2.1) renders the original treatment of Connor Hastie and Taylor extremely difficult. The problem has been studied by Connor et al. (1984) by means of an asymptotic matching procedure. Here the authors consider the case $\omega-\omega_{p i} \ll \omega_{A}$, and solve Eq. (2.1) in two asymptotic regions: one defined by $\hat{s} \theta \sim 1$, the other $\hat{s} \theta \sim \omega_{A}^{2} / \omega\left(\omega-\omega_{p i}\right) \gg 1$. Asymptotic matching of the two solutions then provides a stability criterion that incorporates some diamagnetic effects. The authors also notice that, for Eq. (2.1), a necessary condition for $\omega$ to be imaginary in that $\Re[\omega]=\omega_{p i} / 2$. 
This implies that, at marginality, $\omega\left(\omega-\omega_{p i}\right)=-\omega_{p i}^{2} / 4$, and, more importantly, Eq. (2.1) is solved in an asymptotic expansion in $\omega_{p i}^{2} / 4 \omega_{A}^{2} \ll 1$. Explicitly, we have

$$
k_{2}^{2} \frac{\rho_{i}^{2}}{a L_{p}} \beta_{i} \ll 16 \frac{L_{p}}{a},
$$

which, for a given $\beta_{i}$, determines the range of wavelength for which the $\omega-\omega_{p i} \ll \omega_{A}$ analysis of Eq. (2.1) is valid

$$
\sqrt{\beta_{i}} k_{2} \rho_{i} \ll 4 L_{p} .
$$

We consider this limit to apply and proceed order by order.

Then, to order $z^{\alpha+2}$, one obtains

$$
\frac{d}{d \theta} \frac{g_{N}^{s s}}{\sqrt{g_{N}}} \frac{B_{a}^{2}}{B^{2}} \frac{d g_{0}}{d \theta}=0
$$

and $g_{0}=1$. To order $z^{\alpha+1}$, we have

$$
\frac{d}{d \theta}\left[\frac{g_{N}^{s s}}{\sqrt{g_{N}}} \frac{B_{a}^{2}}{B^{2}}\left(\frac{d g_{1}}{d \theta}+\alpha \hat{s}\right)-\frac{v_{t h i}^{2}}{a L_{p} \omega_{A}^{2}} \frac{a B_{a}^{2}}{P^{\prime}(s)} \frac{j_{\|}}{B}\right]=0 .
$$

A constant of integration is chosen so that $\int_{\Gamma} d \theta d g_{1} / d \theta=0$. Then

$$
\begin{aligned}
& \frac{d g_{1}}{d \theta}+\alpha \hat{s}=\frac{v_{t h i}^{2}}{a L_{p} \omega_{A}^{2}} \frac{a B_{a}^{2}}{P^{\prime}(s)} \frac{\sqrt{g_{N}}}{g_{N}^{s s}} \frac{B^{2}}{B_{a}^{2}} \frac{j_{\|}}{B} \\
& +\frac{\sqrt{g_{N}}}{g_{N}^{s s}} \frac{B^{2}}{B_{a}^{2}} \frac{\alpha \hat{s}-\frac{v_{t h i}^{2}}{a L_{p} \omega_{A}^{2}} \frac{a B_{a}^{2}}{P^{\prime}(s)} \int_{\Gamma} d \theta \frac{\sqrt{g_{N}}}{g_{N}^{s s}} \frac{B^{2}}{B_{a}^{2}} \frac{j_{\|}}{B}}{\int_{\Gamma} d \theta \frac{\sqrt{g_{N}}}{g_{N}^{s s}} \frac{B^{2}}{B_{a}^{2}}},
\end{aligned}
$$

where each term is of the form of those of Eq. (43) of Connor et al. (1979).

To order $z^{\alpha}$, after integrating in $\int_{\Gamma} d \theta$, we obtain

$$
\begin{aligned}
& (\alpha+1) \hat{s} \int_{\Gamma} d \theta \frac{g_{N}^{s s}}{\sqrt{g_{N}}} \frac{B_{a}^{2}}{B^{2}}\left(\frac{d g_{1}}{d \theta}+\alpha \hat{s}\right) \\
& +\frac{v_{t h i}^{2}}{a L_{p} \omega_{A}^{2}} \int_{\Gamma} d \theta\left[\frac{B_{s}}{B} \frac{\partial}{\partial \theta} \frac{B_{a}^{2}}{B^{2}}+\sqrt{g_{N}}\left(2 \frac{P^{\prime}(s)}{B^{2}}+\frac{\partial_{s} B^{2}}{B^{2}}\right)\right]+\frac{v_{t h i}^{2}}{a L_{p} \omega_{A}^{2}} \frac{a B_{a}^{2}}{P^{\prime}\left(s_{0}\right)} \int_{\Gamma} d \theta \frac{j_{\|}}{B} \frac{d g_{1}}{d \theta}=0
\end{aligned}
$$

and, again, each term of this equation resembles those of Eq. (44) of Connor et al. (1979). After using Eq. (2.12), one gets the indicial equation $\alpha(\alpha+1)+\mathcal{D}=0$, with

$$
\begin{aligned}
& \mathcal{D}=\frac{v_{t h i}^{2}}{L_{p} a \omega_{A}^{2} \hat{s}^{2}}\left\{\left(\int_{\Gamma} d \theta \frac{\sqrt{g_{N}} B^{2}}{g_{N}^{s s} B_{a}^{2}}\right) \int_{\Gamma} d \theta\left[\frac{B_{s}}{B} \frac{\partial}{\partial \theta} \frac{B_{a}^{2}}{B^{2}}+\sqrt{g_{N}}\left(2 \frac{P^{\prime}(s)}{B^{2}}+\frac{\partial_{s} B^{2}}{B^{2}}\right)\right]+\right. \\
& \left.\frac{v_{t h i}^{2}}{L_{p} a \omega_{A}^{2}}\left(\frac{a B_{a}^{2}}{P^{\prime}\left(s_{0}\right)}\right)^{2}\left[\left(\int_{\Gamma} d \theta \frac{\sqrt{g_{N}} B^{2}}{g_{N}^{s s} B_{a}^{2}}\right) \int_{\Gamma} d \theta \frac{\sqrt{g_{N}} B^{2}}{g_{N}^{s s} B_{a}^{2}}\left(\frac{j_{\|}}{B}\right)^{2}-\left(\int_{\Gamma} d \theta \frac{\sqrt{g_{N}} B^{2}}{g_{N}^{s s} B_{a}^{2}} \frac{j_{\|}}{B}\right)^{2}\right]\right\} \\
& -2 \frac{v_{t h i}^{2}}{a L_{p} \omega_{A}^{2} \hat{s}} \frac{a B_{a}^{2}}{P^{\prime}(s)}\left[\left(\int_{\Gamma} d \theta \frac{\sqrt{g_{N}}}{g_{N}^{s s}} \frac{B^{2}}{B_{a}^{2}}\right) \int_{\Gamma} d \theta \frac{j_{\|}}{B}-\int_{\Gamma} d \theta \frac{\sqrt{g_{N}}}{g_{N}^{s s}} \frac{B^{2}}{B_{a}^{2}} \frac{j_{\|}}{B}\right] .
\end{aligned}
$$

This implies that $\mathcal{D}=\mathcal{O}(1)$ for

$$
\hat{s} \sim \beta_{i} \frac{a}{L_{p}} \sim \beta^{\prime} \ll 1,
$$


which is the condition that determines the ordering of the global shear for which the asymptotic form (2.7) is acceptable. The Mercier criterion that can be used for comparisons with flux-tube gyrokinetics in a stellarator, when conditions (2.9) and (2.15) apply, is then

$$
\mathcal{D}<1 / 4
$$

for stability, where $\mathcal{D}$ is defined by Eq. (2.14). This result is not new, as each term of Eqs. (2.12) and (2.13) can be identified with the respective terms in Eqs. (43) and (44) of Connor et al. (1979), where different co-ordinates were used. In the absence of equilibrium parallel current, it is a limiting condition on the gradient of the plasma $\beta$ plus a correction due to the covariant component of the equilibrium magnetic field. The usefulness of our result resides in its possible application to gyrokinetic numerical studies, which is now viable since we expressed the stability parameter $\mathcal{D}$ in terms of modified Boozer co-ordinates that commonly interface stallarator equilibria codes and gyrokinetic codes (Xanthopoulos et al. 2009). As a final remark, we notice the relation of our result, derived in modified Boozer co-ordinates, and the common concept of "magnetic well". Since the plasma volume enclosed in a magnetic surface is $V(s)=\int_{0}^{s} d s \int_{0}^{2 \pi} d \theta \int_{0}^{2 \pi} d \zeta \sqrt{g_{N}}$, we have $d^{2} V / d s^{2}=\int_{0}^{2 \pi} d \theta \int_{0}^{2 \pi} d \zeta \partial_{s} \sqrt{g_{N}}$. Had we expressed the curvature drive in Eq. (2.4) in terms of equilibrium poloidal and toroidal current fluxes, we would have been left with the non-secular component of the magnetic drift, $\left(\omega_{\kappa} \omega_{p}\right)_{N S}$, proportional to

$$
\left(\omega_{\kappa} \omega_{p}\right)_{N S} \propto \sqrt{g} B \nabla_{\|}\left(\frac{B_{s}}{B^{2}}\right)+\sqrt{g} \frac{P^{\prime}(s)}{B^{2}}+\frac{1}{B^{2}}\left[J \Psi^{\prime \prime}-I \Phi^{\prime \prime}\right]-\partial_{s} \sqrt{g},
$$

where, indeed, $J$ and $I$ are the toroidal and poloidal current fluxes and $\Phi$ and $\Psi$ are the toroidal and poloidal magnetic fluxes. This expression would replace the non-secular term at the second line of Eq. (2.6), and would result in an explicit dependence on $d^{2} V / d s^{2}$ for the Mercier index in Eq. (2.14). For negative pressure gradients, a positive $d^{2} V / d s^{2}$ then adds to the drive of pressure-driven instabilities making them more unstable. Similarly, a negative $d^{2} V / d s^{2}$ has a stabilising effect (Johnson \& Greene 1967). In the first case, the magnetic configuration is said to possess a "magnetic hill", while is the second case it has a "magnetic well". Even if this nomenclature is somewhat intuitive, our expression seems more conclusive for what concerns the positive-definiteness of the driving terms [the radial derivatives at the first line of Eq. (2.14)]: $\sqrt{g_{N}}\left(2 P^{\prime}(s)+\partial_{s} B^{2}\right) / B^{2}$. From this it is evident that a minimisation of the volume averaged $B^{2}$ is beneficial. The same conclusion was drawn by Boozer (1981) [see discussion after Eq. (29) ]. We conclude this section by noticing that our expression for the Mercier index $\mathcal{D}$ in Eq. (2.14) agrees with Eq. (85) of Cooper (1992), only if the global shear is ordered to be as small as the equilibrium plasma pressure gradient. It is easy to see that this is imposed by the smallness of the global shear. The reason why the global shear has to be small, in our multiple scale asymptotic analysis of the ballooning equation, is explained well in the Introduction of Section II of Connor et al. (1984). While the ballooning equation used to derive the Mercier index of Cooper (1992) does not agree with our starting point [however, see also the alternative improved version of Cooper et al. (1996)], its application to ideal marginal stability is valid and agrees with our result.

\section{Surface-global diamagnetism}

Equation (2.6) implies that, in a local flux-tube, a necessary condition for instability is $\Re[\omega]=\omega_{p i} / 2$. This can be seen by multiplying the equation by the complex conjugate eigenfunction $\psi^{*}$ and integrating by parts along the field line. The result is a second order 
algebraic equation for the eigenvalue, $\omega$, whose imaginary part is not zero only if, indeed, $\Re[\omega]=\omega_{p i} / 2$. We now see how this changes in a surface-global setting. The Laplacian in curvilinear co-ordinates of Eq. (2.2) is more tractable if the metric elements are slowly varying in $x^{2}: \partial_{x^{2}} \ln g_{s s} \sim \partial_{x^{2}} \ln g_{s \theta} \sim \partial_{x^{2}} \ln g^{s s} \ll \partial_{x^{2}} \ln \psi$, then Eq. (2.1) becomes

$$
\frac{1}{\sqrt{g_{B}}} \frac{\partial}{\partial \theta} \frac{B_{a}^{2}}{B^{2} \sqrt{g_{B}}} \frac{\partial}{\partial \theta} \Omega=-\frac{B_{a}^{2}}{B^{2}} \frac{\omega\left(\omega-\omega_{p i}\right)}{\omega_{A}^{2}} \Omega-\frac{v_{t h i}^{2}}{L_{p} a \omega_{A}^{2}} \frac{B}{B_{a}}\left[K_{A S}(\theta)+\epsilon_{h} K_{h}\left(\theta, x^{2}\right)\right] \Omega,
$$

where $\Omega=\Omega_{r}+i \Omega_{i} \equiv \partial_{x^{2}}^{2} \psi$ and the magnetic drift is formally split into an axisymmetric, $K_{A}$, and a non-axisymmetric component $K_{h}$, where $\epsilon_{h}$ is a constant. If we multiply by $\Omega^{*}$, and integrate in $d \theta$, we obtain a quadratic equation for $\omega$ :

$$
\omega^{2}+i \zeta \omega_{p i}^{(0)} \omega+\lambda^{2}=0
$$

with

$$
\begin{gathered}
\zeta=\zeta_{r}+i \zeta_{i} \equiv \frac{\oint d x^{2} \int_{-\infty}^{\infty} d \theta \frac{\sqrt{g_{B}} B_{a}^{2}}{B^{2}} \Omega^{*} \partial_{x^{2}} \Omega}{\oint d x^{2} \int_{-\infty}^{\infty} d \theta \frac{\sqrt{g_{B}} B_{a}^{2}}{B^{2}}|\Omega|^{2}} \\
\lambda^{2}=\frac{v_{t h i}^{2}}{L_{p} a} \frac{\oint d x^{2} \int_{-\infty}^{\infty} d \theta \frac{\sqrt{g_{B}} B_{a}^{2}}{B^{2}}\left[K_{A S}(\theta)+\epsilon_{h} K_{h}\left(\theta, x^{2}\right)\right]|\Omega|^{2}}{\oint d x^{2} \int_{-\infty}^{\infty} d \theta \frac{\sqrt{g_{B}} B_{a}^{2}}{B^{2}}|\Omega|^{2}} \\
-\omega_{A}^{2} \frac{\oint d x^{2} \int_{-\infty}^{\infty} d \theta \frac{\sqrt{g_{B}} B_{a}^{2}}{B^{2}}\left|\frac{\partial \Omega}{\partial \theta}\right|^{2}}{\oint d x^{2} \int_{-\infty}^{\infty} d \theta \frac{\sqrt{g_{B}} B_{a}^{2}}{B^{2}}|\Omega|^{2}}
\end{gathered}
$$

and

$$
\omega_{p i}^{(0)}=\frac{1}{2} \frac{\rho_{i}}{a} \frac{v_{t h i}}{L_{n}}\left(1+\eta_{i}\right) .
$$

In the strongly driven case, $\lambda \gg|\zeta| \omega_{p i}^{(0)}$, the ideal MHD growth rate and a small real correction are found

$$
\omega \approx i \gamma_{M H D}+\frac{\omega_{p i}^{(0)}}{2} \zeta_{i}
$$

The real correction to the ideal MHD growth rate is the frequency $\omega_{p i}^{(0)} / 2$ times a surfaceglobal factor. The result is then

$$
\omega_{r}=\frac{\omega_{p i}^{(0)}}{2} \frac{\oint d x^{2} \int_{-\infty}^{\infty} d \theta \frac{\sqrt{g_{B}} B_{a}^{2}}{B^{2}}\left(\Omega_{r} \partial_{x^{2}} \Omega_{i}-\Omega_{i} \partial_{x^{2}} \Omega_{r}\right)}{\oint d x^{2} \int_{-\infty}^{\infty} d \theta \frac{\sqrt{g_{B}} B_{a}^{2}}{B^{2}}|\Omega|^{2}} .
$$

Let us now consider a trial function which is a rotation by an angle $k_{2} x^{2}$ of a function $\hat{\Omega}(\theta)$ defined on a flux-tube

$$
\Omega\left(\theta, x^{2}\right)=\hat{\Omega}(\theta)\left[\cos \left(k_{2} x^{2}\right)+i \sin \left(k_{2} x^{2}\right)\right],
$$

Eq. (3.5) then reduces to the local result

$$
\omega_{r}=\frac{\omega_{p i}}{2} \equiv \frac{1}{4} \frac{v_{t h i}}{L_{n}}\left(1+\eta_{i}\right) k_{2} \frac{\rho_{i}}{a} .
$$

A less trivial field-line-label dependence of the eigenfunction generates an effective surface-global diamagnetic frequency. Let us consider, for instance, a system with helical 
symmetry, thus

$$
\Omega\left(\theta, x^{2}\right)=\sum_{l=-M}^{M} \hat{\Omega}_{l}(\theta)\left\{\cos \left[l\left(q \theta+x^{2}\right)\right]+i \sin \left[l\left(q \theta+x^{2}\right)\right]\right\} .
$$

The contribution to the surface-global real frequency of each helical harmonic $M$ is proportional to

$$
\Omega_{r} \partial_{x^{2}} \Omega_{i}-\Omega_{i} \partial_{x^{2}} \Omega_{r}=M\left\{\cos ^{2}\left[M\left(q \theta+x^{2}\right)\right]+\sin ^{2}\left[M\left(q \theta+x^{2}\right)\right]\right\}=M,
$$

thus

$$
\omega_{r}^{(M)}=\frac{\omega_{p i}^{(0)}}{2} M
$$

and the marginal frequency is affected by the number of poloidal turns it takes the helix to close onto itself.

We conclude that, in a surface-global setting, for large pressure gradients, the real frequency of unstable KBMs [as described by diamagnetic MHD, Eq. (2.1)] can differ from the value $\omega_{p i} / 2$ for purely geometrical reasons.

\section{Lattice-drift model for KBMs}

A further geometric effect that we expect to observe is associated with the $x^{2}$-dependence of the strength of the curvature drive, the term that multiplies $\omega_{\kappa}$ in Eq (2.1). In Eq. (3.1), this term was formally separated into an axisymmetric and a non-axisymmetric part: $\omega_{\kappa} \propto K_{A S}(\theta)+\epsilon_{h} K_{h}\left(\theta, x^{2}\right)$. The effect of the $x^{2}$ dependence in $\omega_{\kappa}$ has been investigated for the case of the ion-temperature-gradient mode. In the work of Zocco et al. (2016), the authors performed an asymptotic expansion in $\epsilon_{h} \ll 1$. For finite $\epsilon_{h}$, the authors introduced a discrete Fourier expansion of the ITG eigenvalue equation (Zocco et al. 2018). The non-axisymmetric term $\epsilon_{h} K_{h}\left(\theta, x^{2}\right)$ then generates a side-band coupling of the Fourier component of the eigenfunction. The eigenvalue equation is written in a matrix form, and a surface-global eigenvalue equation is given by setting to zero the determinant of the matrix, which strikingly resembles the equation of state of quantum electrons in a periodic crystal. The same approach is now possible for KBMs, however there is now a complication owing to the second order derivative on the LHS of Eq. (2.1), which was neglected in the aforementioned ITG studies. In practice, we need to introduce an explicit form for $\omega_{\kappa}$ is Eq. (2.1), expand the eigenfunction using as a basis the functions used to construct $\omega_{\kappa}$, and study a system of coupled ballooning equations, rather than one ballooning equation, which is sufficient in the axisymmetric case, since $\omega_{\kappa}$ is a function of $\theta$ only. The careful reader might recognise that such approach is similar to the flux-tube-bundle model introduced by Sugama et al. (2012) and used numerically by Nunami et al. (2010). Thus, we proceed by neglecting the complications related to the $x^{2}$ dependence of the LHS of Eq. (2.1), and start with Eq. (3.1). We assume $B^{2} \approx B_{a}^{2}$ and take $\sqrt{g_{B}}=$ const. We add a small helical correction to the driving term found in concentric circular geometry

$$
\begin{aligned}
& K_{A S}(\theta)+\epsilon_{h} K_{h}\left(\theta, x^{2}\right)=\cos \theta+\hat{s} \theta \sin \theta \\
& +\epsilon_{h}\left\{\cos \left[M\left(q \theta+x^{2}\right)\right]+\sin \left[M\left(q \theta+x^{2}\right)\right]\right\},
\end{aligned}
$$

where $L_{B}$ is some effective average radius of curvature. The system is artificial but useful to build up some intuition to be used in the interpretation of either surface-global or flux-bundle numerical simulations. If we use $k_{2} \rightarrow-i \partial_{x^{2}}, \psi=\sum_{m} \psi_{m} \exp \left[2 \pi x^{2} / a\right]$, Eq. 
(3.1) becomes

$$
\begin{aligned}
& \frac{1}{\beta_{i}} \frac{\partial}{\partial \theta}\left(1+\hat{s}^{2} \theta^{2}\right) \frac{\partial}{\partial \theta} \psi_{m}=-\hat{\omega}\left[\hat{\omega}-\frac{\ell_{c}}{2 L_{n}} \rho_{*} m\left(1+\eta_{i}\right)\right]\left(1+\hat{s}^{2} \theta^{2}\right) \psi_{m} \\
& -\frac{\ell_{c}^{2}}{L_{p} L_{B}}(\cos \theta+\hat{s} \theta \sin \theta) \psi_{m} \\
& -\epsilon_{h} \frac{\ell_{c}^{2}}{2 L_{p} L_{B}}\left\{e^{i M q \theta}\left(1-\frac{M}{m}\right)^{2} \psi_{m-M}+e^{i M q \theta}\left(1+\frac{M}{m}\right)^{2} \psi_{m+M}\right\},
\end{aligned}
$$

where $\hat{\omega}=\omega /\left(v_{t h i} / \ell_{c}\right), \ell_{c}$ is a connection length, and $L_{B}$ an effective radius of curvature. The first two lines of Eq. (4.2) are simply the Fourier series expansion of the axisymmetric equation studied by Aleynikova \& Zocco (2017). Non-axisymmetry is induced by the helical term. Let us consider a given $m_{0} \sim \rho_{*}^{-1} \gg 1, m=m_{0}-\Delta m$ and $\hat{\omega}=\hat{\omega}_{0}+i \hat{\gamma}_{0}+$ $\delta \hat{\omega} \equiv \bar{\omega}+\delta \hat{\omega}$, where

$$
\frac{\Delta m}{m_{0}} \sim \frac{\delta \hat{\omega}}{\left|\hat{\omega}^{(0)}\right|} \sim \epsilon_{h} \ll 1,
$$

with $\hat{\omega}_{0}=\left(\ell_{c} / 4 L_{n}\right) m_{0} \rho_{*}\left(1+\eta_{i}\right)$ and $\hat{\gamma}_{0}=\Im[\bar{\omega}]$, where $\bar{\omega}$ is the solution of the quadratic equation for the axisymmetric problem $\bar{\omega}\left(\bar{\omega}-\omega_{p i}\right)+\tilde{\lambda}^{2}=0$, and

$$
\tilde{\lambda}^{2}=\frac{\ell_{c}^{2}}{L_{p} L_{B}} \frac{\int_{-\infty}^{\infty} d \theta(\cos \theta+\hat{s} \theta \sin \theta)\left|\psi_{m_{0}}\right|^{2}}{\int_{-\infty}^{\infty} d \theta\left(1+\hat{s}^{2} \theta^{2}\right)\left|\psi_{m_{0}}\right|^{2}}-\frac{1}{\beta_{i}} \frac{\int_{-\infty}^{\infty} d \theta\left(1+\hat{s}^{2} \theta^{2}\right)\left|\frac{\partial \psi_{m_{o}}}{\partial \theta}\right|^{2}}{\int_{-\infty}^{\infty} d \theta\left(1+\hat{s}^{2} \theta^{2}\right)\left|\psi_{m_{0}}\right|^{2}} .
$$

Notice that in the subsidiary $\rho_{*} m_{0} \ll 1$ limit the mode is purely growing and $\psi_{m}$ is real. We now consider this limit. After using the finite-difference formula for the $m$-space derivatives, the imaginary part of the first order correction reads

$$
\Im[\delta \hat{\omega}]=\epsilon_{h} \frac{\ell_{c}^{2}}{L_{p} L_{B} \hat{\gamma}_{0}} \frac{\int_{-\infty}^{\infty} d \theta \cos (q \theta)\left|\psi_{m_{0}}\right|^{2}\left(\left.\frac{2}{\psi_{m_{0}}} \frac{\partial^{2} \psi}{\partial m^{2}}\right|_{m_{0}}-1\right)}{\int_{-\infty}^{\infty} d \theta\left(1+\hat{s}^{2} \theta^{2}\right)\left|\psi_{m_{0}}\right|^{2}},
$$

which is finite for $\rho_{*} m_{0} \ll 1$, and always negative if $\psi_{m}$ has a maximum in $m_{0}$. This result proves that the helical correction to the axisymmetric ballooning mode is stabilising. Perhaps, the most important feature of Eq. (4.4) is that stabilisation occurs for any value of $q$, while the Mercier condition for stability, for concentric circular cross sections, shows a strong dependence on $q$ (Glasser et al. 1976; Porcelli \& Rosenbluth 1998)

$$
\mathcal{D}=\frac{8 \pi r}{\hat{s}^{2} B}\left|\frac{d p}{d r}\right|\left(1-q^{2}\right)<\frac{1}{4} .
$$

Equation (4.4) then implies that a system can be ballooning unstable according to the Mercier criterion, but the surface global effect could mitigate the instability.

\section{Summary and Discussion}

In this article we studied several new aspects of kinetic ballooning modes in magnetically confined toroidal plasmas that stem from purely geometric properties of the confining magnetic field. This was done for large equilibrium plasma pressure gradients since, in this limit, analytical progress can be made. The surface-global formulation of the problem was presented. Here, physical quantities are kept radially local but variations in the field-line-label co-ordinate are allowed for both equilibrium and perturbed fields. A 
novel form of the Mercier stability criterion, useful for quantitative comparison with stellarator flux-tube gyrokinetic codes was given. The use of modified Boozer co-ordinates led us to the conclusion that a minimization of the average of the magnetic field magnitude square is beneficial for stability. We explain the relation between this result and the stabilizing effect of magnetic wells on equilibrium configurations. For surface-global systems, we derived the general form equivalent to the necessary condition for instability of KBMs which constraints the frequency of the mode. It is found that purely geometric effects can result in mode frequencies that differ from the tokamak resul $\Re[\omega]=\omega_{p i} / 2$, where $\omega_{p i}$ is the total diamagnetic frequency of the ions. Finally, the effect of the coupling of several flux tubes covering a flux surface has been studied. This coupling has a stabilising effect on the local most unstable mode, and can lead to a possible violation of the Mercier criterion.

We thank Per Helander, Jürgen Nührenberg, Jack Connor, Hideo Sugama, Masanori Nunami and Motoki Nakata, for their comments on different aspects of this work. Part of this work was motivated by discussions with Michele Romanelli in the framework of the ITPA for transport and confinement, and was carried out under the auspices of EUROfusion for the JET task T17-05 D3 "Gyrokinetic simulation in the pedestal (global effects)" and was completed during a visit at the National Institute for Fusion Science, Toki, Gifu, Japan. We thank Fulvio Zonca and Alexey Mishchenko for their comments on the work on kinetic ballooning mode theory presented by KA at the 17th European Fusion Theory Conference, Athens, October 2017.

\section{REFERENCES}

Aleynikova, Ksenia \& Zocco, Alessandro 2017 Quantitative study of kinetic ballooning mode theory in simple geometry. Phys. Plasmas 24 (9), 092106.

Aleynikova, K., Zocco, A., Xanthopoulos, P., Helander, P. \& Nührenberg, C. 2018 Kinetic ballooning modes in tokamaks and stellarators. Journal Plasma Phys. To appear on this issue.

Bauer, F., Betancourt, O. \& Garabedian, Paul 1984 Magnetohydrodynamic Equilibrium and Stability of Stellarators. Springer-Verlag.

Baumgaertel, J. A., Hammett, G. W., Mikkelsen, D. R., Nunami, M. \& XanthopouLOS, P. 2012 Gyrokinetic studies of the effect of $\beta$ on drift-wave stability in the national compact stellarator experiment. Phys. Plasmas 19 (12), 122306.

Boozer, Allen H. 1981 Plasma equilibrium with rational magnetic surfaces. Phys. Fluids 24 (11), 1999-2003.

Boozer, Allen H. 1982 Establishment of magnetic coordinates for a given magnetic field. The Physics of Fluids 25 (3), 520-521.

Chen, Liu \& Zonca, Fulvio 2016 Physics of Alfvén waves and energetic particles in burning plasmas. Rev. Mod. Phys. 88, 015008.

Connor, J.W., TAng, W.M. \& Allen, L. 1984 Finite-Larmor-Radius modification of the Mercier criterion. Nucl. Fusion 24 (8), 1023.

Connor, J W, Hastie, R J \& Taylor, J B 1978 Phys. Rev. Lett. 40 (6), 396.

Connor, J. W., Hastie, R. J. \& Taylor, J. B. 1979 High mode number stability of an axisymmetric toroidal plasma. Proceedings of the Royal Society of London A: Mathematical, Physical and Engineering Sciences 365 (1720), 1-17.

Cooper, A 1992 Variational formulation of the linear MHD stability of 3D plasmas with noninteracting hot electrons. Plasma Phys. Control. Fusion 34 (6), 1011.

Cooper, W. A., Singleton, D. B. \& Dewar, R. L. 1996 Spectrum of ballooning instabilities in a stellarator. Phys. Plasmas 3 (1), 275-280.

Correa-Restrepo, D. 1978 Ballooning modes in three-dimensional MHD equilibria. Z. Naturforsch. 33a, 789-791.

Faber, B. J., Pueschel, M. J., Proll, J. H. E., Xanthopoulos, P., Terry, P. W., 
Hegna, C. C., Weir, G. M., Likin, K. M. \& Talmadge, J. N. 2015 Gyrokinetic studies of trapped electron mode turbulence in the helically symmetric experiment stellarator. Phys. Plasmas 22 (7), 072305.

Fu, G. Y., Cooper, W. A., Gruber, R., Schwenn, U. \& Anderson, D. V. 1992 Fully three-dimensional ideal magnetohydrodynamic stability analysis of low- $n$ modes and Mercier modes in stellarators. Phys. Fluids B: Plasma Physics 4 (6), 1401-1411.

Gardner, H.J. \& Blackwell, D.B. 1992 Calculation of mercier stability limits of toroidal heliacs. Nucl. Fusion 32 (11), 2009.

Glasser, A. H., Green, J. M. \& Johnson, J. L. 1976

Zocco, A., Xanthopoulos, P. \& Helander, P. 2018 Geometric stabilization of the electrostatic ion-temperature-gradient driven instability. II. non-axisymmetric systems. To be submitted. Phys. Fluids. 19 (4), 567.

Hamada, S. 1962 Hydromagnetic equilibria and their proper coordinates. Nucl. Fusion 2, 23.

Hastie, R.J. \& TAYlor, J.B. 1981 Validity of ballooning representation and mode number dependence of stability. Nucl. Fusion 21 (2), 187.

Hegna, C. C. \& Nakajima, N. 1998 On the stability of mercier and ballooning modes in stellarator configurations. Phys. Plasmas 5 (5), 1336-1344.

Helander, P., Bird, T., Jenko, F., Kleiber, R., Plunk, G.G., Proll, J.H.E., Riemann, J. \& Xanthopoulos, P. 2015 Advances in stellarator gyrokinetics. Nucl. Fusion 55 (5), 053030 .

Ishizawa, A., Watanabe, T.-H., Sugama, H., Maeyama, S. \& Nakajima, N. 2014 Electromagnetic gyrokinetic turbulence in finite-beta helical plasmas. Phys. Plasmas 21 (5), 055905 .

Ishizawa, A., Watanabe, T.-H., Sugama, H., Nunami, M., Tanaka, K., Maeyama, S. \& NAKAJima, N. 2015 Turbulent transport of heat and particles in a high ion temperature discharge of the Large Helical Device. Nucl. Fusion 55 (4), 043024.

Jenko, F \& KendL, A 2002 Radial and zonal modes in hyperfine-scale stellarator turbulence. Phys. Plasmas 9 (10), 4103.

Johnson, J L \& Greene, J M 1967 Resistive interchanges and the negative $V^{\prime \prime}$ criterion. Plasma Phys. 9 (5), 611.

Mercier, C 1960 Un critere necessaire de stabilité hydromagnetic pour un plasma en symetry de revolution. Nucl. Fusion 1, 47-53.

Mercier, C. \& Luc, H. 1974 Lectures in Plasma Physics. Commision of the European Communities.

Mishchenko, A., Borchardt, M., Cole, M., Hatzky, R., Fehér, T., Kleiber, R., KöNies, A. \& ZocCO, A. 2015 Global linear gyrokinetic particle-in-cell simulations including electromagnetic effects in shaped plasmas. Nucl. Fusion 55 (5), 053006.

Nührenberg, J. \& Zille, R. 1987 Equilibrium and stability of low-shear stellarators. Theory of Fusion Plasmas pp. 3-23.

Nunami, Masanori, Watanabe, Tomo-Hiko \& Sugama, Hideo 2010 Gyrokinetic Vlasov code including full three-dimensional geometry of experiments. Plasma Fus. Research 5, 016-016.

Plunk, G. G., Helander, P., Xanthopoulos, P. \& Connor, J. W. 2014 Phys. Plasmas $21(3)$.

Porcelli, F \& Rosenbluth, M N 1998 Modified mercier criterion. Plasma Phys. Control. Fusion 40 (4), 481.

Proll, J. H. E., Xanthopoulos, P. \& Helander, P. 2013 Collisionless microinstabilities in stellarators. ii. numerical simulations. Phys. Plasmas 20 (12), 122506.

Roberts, K. V. \& Taylor, J. B. 1965 Phys. Fluids 6 (2), 315-322.

Sugama, H. \& Watanabe, T.-H. 2004 Study of electromagnetic microinstabilities in helical systems with the stellarator expansion method. Phys. Plasmas 11 (6), 3068-3077.

Sugama, H., Watanabe, T.-H., Nunami, M., Satake, S., Matsuoka, S. \& Tanaka, K 2012 Kinetic simulations of neoclassical and anomalous transport processes in helical systems. Plasma and Fusion Research 7, 2403094.

Tang, W.M., Connor, J.W. \& Hastie, R.J. 1980 Nucl. Fusion 20 (11), 1439.

Xanthopoulos, P., Cooper, W. A., Jenko, F., Turkin, Yu., Runov, A. \& Geiger, 
J. 2009 A geometry interface for gyrokinetic microturbulence investigations in toroidal configurations. Phys. Plasmas 16 (8).

Xanthopoulos, P., Plunk, G. G., Zocco, A. \& Helander, P. 2016 Phys. Rev. X 6, 021033.

Zocco, A., Plunk, G. G., Xanthopoulos, P. \& Helander, P. 2016 Geometric stabilization of the electrostatic ion-temperature-gradient driven instability. I. Nearly axisymmetric systems. Phys. Plasmas 23 (8).

Zocco, A., Xanthopoulos, P. \& Helander, P. 2018 Geometric stabilization of the electrostatic ion-temperature-gradient driven instability. II. non-axisymmetric systems. To be submitted 